удК 621.313 .332

\title{
ИССЛЕДОВАНИЕ АСИНХРОННОЙ ЭЛЕКТРИЧЕСКОЙ МАШИНЫ С КОРОТКОЗАМКНУТЫМ РОТОРОМ ДЛЯ ВОЗОБНОВЛЯЕМОЙ ЭНЕРГЕТИКИ ПРИ НЕСТАЦИОНАРНОМ КОНДЕНСАТОРНОМ ВОЗБУЖДЕНИИ
}

\author{
Буньков Дмитрий Сергеевич1, \\ bunkovds@tpu.ru
}

\author{
Глазырин Александр Савельевич2,3, \\ asglazyrin@tpu.ru
}

\section{Боловин Евгений Владимирович2, orange@tpu.ru}

\section{Крохта Юрий Владимирович1,} yuriy.krokhta@gmail.com

\section{Баннов Дмитрий Михайлович4, bannov.dm@samgtu.ru}

\author{
Ковалев Владимир Захарович ${ }^{3}$, \\ vz_kovalev@mail.ru
}

\author{
Хамитов Рустам Нуриманович5,6, \\ apple_27@list.ru
Кладиев Сергей Николаевич², kladiev@tpu.ru
Ланграф Сергей Владимирович ${ }^{7}$, sergeylgrf@gmail.com

\section{Леонов Андрей Петрович2,} leonov_ap@tpu.ru

1000 «Элетим», Россия, 634021, г. Томск, ул. Алтайская, 161А.

2 Национальный исследовательский Томский политехнический университет, Россия, 634050, г. Томск, пр. Ленина, 30

3 Югорский государственный университет, Россия, 628012, г. Ханты-Мансийск, ул. Чехова, 16.

4 Самарский государственный технический университет, Россия, 443100, г. Самара, ул. Молодогвардейская, 244.

5 Омский государственный технический университет, Россия, 644050, г. Омск, пр. Мира, 11

6 Тюменский индустриальный университет, Россия, 625000, г. Тюмень, ул. Володарского, 38.

7 ООО «НПФ Мехатроника-Про», Россия, 634021, г. Томск, пр. Фрунзе, 119E.

\begin{abstract}
Актуальность. Доля электроэнергии, вырабатываемой установками на основе возобновляемой энергии, постоянно растет, в связи с чем потребность в развитии систем питания и автоматического управления электрическими машинами, лежащими в основе ветро- и гидрогенераторов, не теряет актуальности. В составе таких генераторных установок переменного тока применяют синхронные электрические машины, асинхронные машины с фразным и короткозамкнутым ротором. Преобразователи частоты, устанавливаемые в статорные и роторные цепи асинхронных машин переменного тока, позволяют управлять процессами их возбуждения, однако для начала генерации требуется использовать дополнительные внешние источники питания. Благодаря остаточному намагничиванию в магнитопроводе можно обеспечить процесс гарантированного самовозбуждения асинхронной машины с короткозамкнутым ротором с помощью подключения батарей конденсаторов к ее статорным обмоткам без применения дополнительного внешнего источника питания. Предложенный способ нестационарного конденсаторного возбуждения позволяет обеспечить адаптацию генераторной установки к изменению режимов работы в условиях децентрализованного электроснабжения.

Цель: исследовать предложенную систему стабилизации напряжения асинхронной машины с короткозамкнутым ротором с варьируемым конденсаторным возбуждением в составе источника возобновляемой энергии.

Методы: теоретические - теория диффференциальных уравнений, методы численного решения обыкновенных диффреренциальных уравнений, теория электропривода, теория электрических машин, численные методы аппроксимации данных, и экспериментальные - проведение испытаний асинхронной электрической машины с нестационарным конденсаторным возбуждением на разработанном испытательном стенде с целью получения нагрузочных характеристик и осциллограмм напряжений на обмотках статора асинхронных машин с короткозамкнутым ротором в различных режимах работы, методы исследования.

Результаты. Разработана и изготовлена оригинальная экспериментальная установка с узлом, имитирующим работу турбины. Электромеханический преобразователь энергии выполнен в виде асинхронной машины с короткозамкнутьм ротором $c$ нестационарным конденсаторным возбуждением. Блок управления установкой выполнен в виде интегрированного с силовым блоком гальванически развязанного модуля во влагостойком исполнении с применением беспроводного интерфрейса связи Bluetooth. Описан и протестирован способ коммутации батарей конденсаторов с применением тиристоров в качестве управляемого ключа с двухсторонней проводимостью. Анализ полученных нагрузочных характеристик показывает принципи-
\end{abstract}


альную возможность обеспечить гарантированную выработку электроэнергии со стабилизацией напряжения в допустимых пределах изменения мощности нагрузки. Примененная в составе экспериментальной установки система автоматической коммутации конденсаторов с варьируемой в зависимости от потребляемой мощности емкостью позволила обеспечить приемлемое время динамической реакции на возмущающее воздействие при нестационарной нагрузке.

\section{Ключевые слова:}

Возобновляемые источники энергии, асинхронная машина, короткозамкнутый ротор, нестационарное конденсаторное возбуждение, децентрализованное электроснабжение.

\section{Введение}

Электромеханические преобразователи энергии на основе асинхронных машин (AM) с короткозамкнутым (КЗ) ротором с конденсаторным возбуждением входят в состав ветроэнергетических установок (ВЭУ) $[1,2]$ и микро-гидроэлектростанций (микро-ГЭС) $[3,4]$.

Широкому распространению и массовому повсеместному внедрению ВЭУ препятствуют такие известные их недостатки, как инфразвуки, шумы, большая стоимость, долгая окупаемость, плохая ремонтопригодность для установок средней и высокой мощности [5-10]. В то же время существуют экономически оправданные районы применения ВЭУ, среди которых на территории РФ в первую очередь стоит отметить центральные районы Горного Алтая [11] и некоторые районы республики Саха (Якутия) [12, 13].

Ветроэнергетические ресурсы для центральных районов Горного Алтая в верхнем поясе гор оцениваются в пределах от 200 до $500 \mathrm{BT} / \mathrm{m}^{2}$ удельной мощности для зон с малой энергетикой, располагающихся на наветренных склонах и водоразделах выше 1500 м над уровнем моря $[11,14]$. Бесперспективными для размещения ВЭУ являются днища межгорных котловин, подветренные склоны и узкие горные долины, ориентированные перпендикулярно преобладающему направлению ветров [15].

Проведенный кластерный анализ республики Саха (Якутия) показал, что существуют также перспективные с точки зрения ветроэнергетики районы с высоким значением потенциала энергии ветра и являющиеся наиболее оправданными для внедрения ветроэнергетических установок $[12,13]$.

Возобновляемые источники энергии (ВИЭ) на основе микро-ГЭС характеризуются относительно высокими первоначальными затратами [16], связанными с адаптацией оборудования к рельефу местности, гидрологическим характеристикам водного источника. Для рек бассейна Томи характерным является высокое значение уклонов и водной поверхности во все фазы водного режима, как в многоводные, так и в маловодные годы [17]. Наибольшей энергией обладают потоки, формирующиеся на западных склонах Кузнецкого Алатау: p. Тутуяс $\left(442 \cdot 10^{6}\right.$ кВт ч/год), р. Тайдон $\left(528 \cdot 10^{6}\right.$ кВт॰ч/год), а также в Горной Шории p. Тельбес $\left(1226 \cdot 10^{6}\right.$ кВт ч/год), что легко объясняется значительным перепадом высот и водности потоков. В современном мире для малой гидроэнергетики рекомендуется использовать поперечно струйные или двукратные гидротурбины [17-19], а также перспективным является применение микро-ГЭС рукавного типа $[16,20]$.
На сегодняшний день наиболее распространены и массово применяются описанные далее типы электротехнических комплексов ВИЭ на основе ВЭУ и микро-ГЭС [21], имеющих свои преимущества и недостатки [22].

К основным преимуществам электротехнического комплекса (ЭТК), включающего синхронную электрическую машину с возбуждением от постоянных магнитов, выпрямитель, DC/DC преобразователь и автономный инвертор напряжения (АИН) [23], относятся:

1) высококачественное возбуждение при применении постоянных магнитов с высокой коэрцитивной силой;

2) отсутствие подвижных токоведущих частей;

3) формирование электронной системой стабилизации высококачественных синусоидальных сигналов в широком диапазоне скоростей ветра;

4) возможность экстремальной настройки по отбору мощности.

5) К недостаткам данного электротехнического комплекса относятся:

6) дополнительное снижение КПД за счет многократного преобразования энергии;

7) высокая стоимость синхронной машины из-за применяемых в ее составе высококачественных магнитных материалов

8) отсутствие возможности регулирования реактивной мощности на низких значениях угловой скорости турбины;

9) необходимость применения повышающего DC/DC преобразователя.

ЭТК на основе синхронной электрической машины с постоянными магнитами являются наиболее универсальными источниками питания для малой возобновляемой энергетики.

Другим распространенным типом является ЭТК на основе АM двойного питания, выпрямителя, DC/DC преобразователя и АИН, подключенного в роторную цепь. Преимущества этого ЭТК:

1) отсутствие повышающего DC/DC преобразователя;

2) высококачественное электромагнитное возбуждение за счет регулирования энергии скольжения с помощью ПЧ, установленного в роторную цепь;

3) отсутствие необходимости в дополнительной фильтрации напряжения, снимаемого со статора, так как АМ выступает в качестве своеобразного фильтра низких частот, подавляющего ШИМ сигнал;

4) приемлемый отбор мощности, осуществляемый в достаточно широком диапазоне частот вращения турбины; 
5) возможность экстремальной настройки системы управления по отбору мощности [24].

Недостатки этого ЭТК:

1) значительное количество потребляемой электроэнергии, расходуемой на собственные нужды генераторной установки;

2) необходимость применения повышающего редуктора;

3) наличие подвижных токоведущих частей.

В области малой автономной энергетики особенностью ЭТК на основе АМ с КЗ ротором с конденсаторными батареями в цепях статора является гарантированное самовозбуждение генератора в режиме холостого хода. Преимущества этого ЭТК:

1) самая надежная и дешевая машина переменного тока, способная выдерживать кратковременные механические и электрические перегрузки;

2) возможность обезопасить подключаемую нагрузку и сам генератор в случае возникновения режимов короткого замыкания, вызывающего срыв генерации напряжения благодаря особенностям конструкции АМ с КЗ ротором;

3) возможность работы установки без дорогостоящего силового преобразователя.
Недостатки этого ЭТК:

1) необходимость поддержания постоянной частоты вращения вала АМ с КЗ ротором;

2) необходимость обеспечения гарантированного процесса самовозбуждения до подключения нагрузки;

3) невозможность обеспечения стабилизации выходного напряжения при изменении нагрузки;

4) вероятность возникновения избыточного механического перенапряжения в рабочем колесе гидротурбины при срыве генерации напряжения.

Рассмотренный в данной статье способ нестационарного конденсаторного возбуждения АМ с КЗ ротором позволяет обеспечить стабилизацию выходного напряжения энергетической установки при изменении нагрузки при постоянной частоте вращения.

Принципы функционирования генераторной установки с нестационарным конденсаторным возбуждением

Предложенный тип генераторной установки для возобновляемой энергетики на базе АМ с КЗ ротором c нестационарным конденсаторным возбуждением представлен на функциональной схеме (рис. 1).

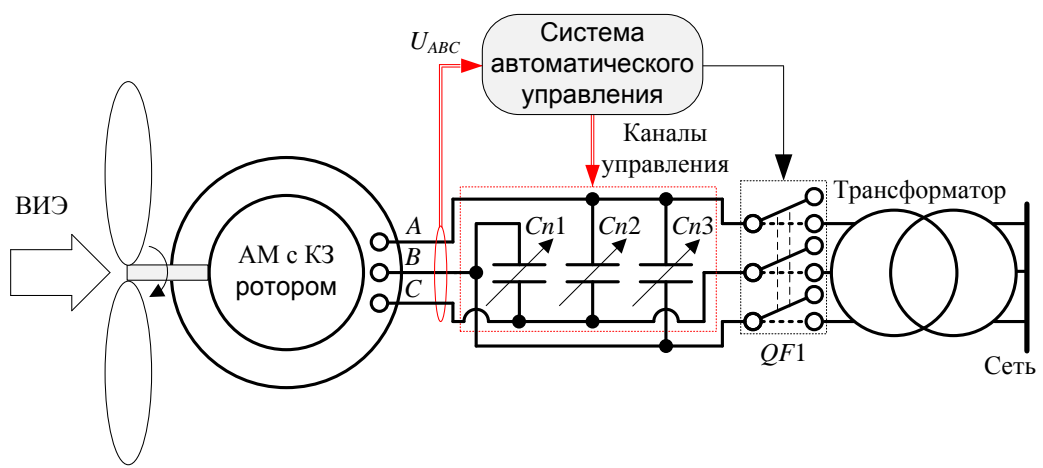

Рис. 1. Функииональная схема генераторной установки на базе АМ с КЗ ротором с нестаиионарным конденсаторным возбуждением

Fig. 1. Functional diagram of squirrel-cage induction generator with non-stationary capacitive excitation

Турбина энергетической установки (рис. 1) приводится во вращение за счет энергии водяного потока или ветра. Важным условием применения предложенной энергетической установки является стабильная частота вращения вала АМ с КЗ ротором, что гарантирует номинальную частоту генерируемого напряжения. Постоянство частоты вращения вала АМ с КЗ ротором для ВЭУ обеспечивается системой аэродинамического регулирования, например, за счет срыва потока воздуха или поворота лопастей. Постоянство напора и расхода воды через турбину микро-ГЭС обеспечивается стабилизацией механического момента на приводном валу электрической машины. Для поддержания номинального уровня частоты генерируемого напряжения кроме этого необходимо обеспечить стабилизацию потребляемой активной мощности. Нестационарное конденсаторное возбуждение обеспечивается тремя регулируемыми конденсаторными батареями, соединенными в треугольник параллельно выводам $A, B, C$ статора AM с КЗ ротором. Контактор $Q F 1$ коммутирует обмотки статора с первичными обмотками трансформатора, работающего на систему децентрализованного электроснабжения, после завершения процесса самовозбуждения АМ с КЗ ротором. Трансформатор обеспечивает гальваническую развязку и согласование цепей энергетической установки и питаемой сети. Система автоматического управления (САУ) регулирует емкость конденсаторных батарей согласно уровню напряжения $U_{A B C}$, измеряемого на выходах $A, B, C$ АМ с КЗ ротором. Подключение конденсаторных батарей по схеме треугольник обусловлено работой на симметричную активную нагрузку. Для той же мощности нагрузки, но при несимметричном ее потреблении, рациональнее применять соединение конденсаторных батарей звездой, что дает возможность независимого регулирования напряжения каждой фазы, однако при этом необходимо применять конденсаторы большей емкости.

Устройство конденсаторной батареи, применяемой в составе генераторной установки (рис. 1), представлено на рис. 2. 


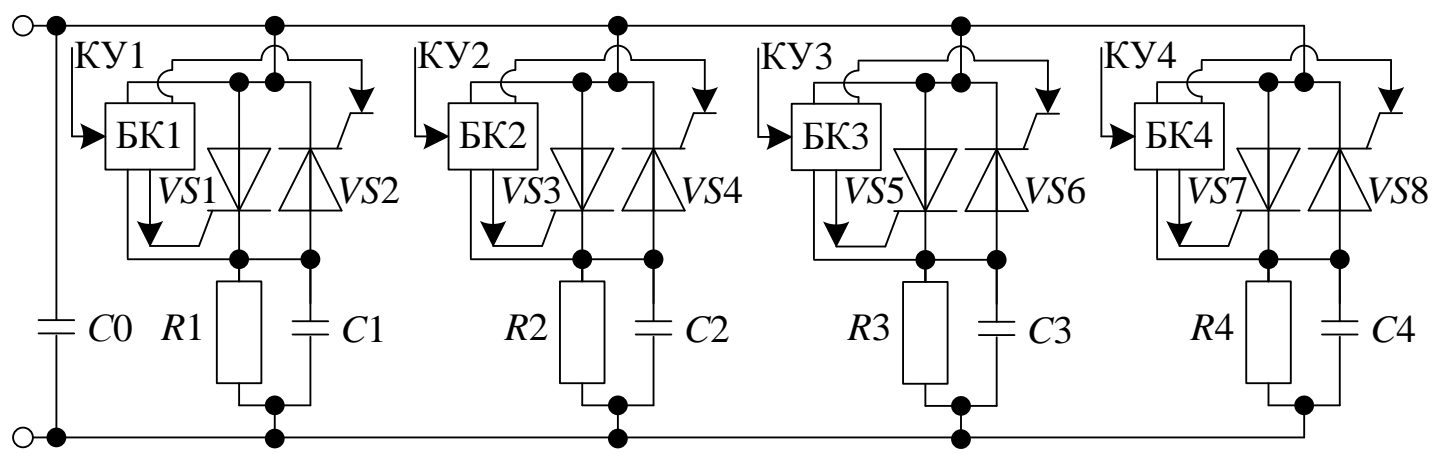

Рис. 2. Схема соединения элементов конденсаторной батареи, включающей четыре коммутируемые конденсаторные стойки

Fig. 2. Schematic diagram of capacitor bank elements, including four switched capacitor branch

В представленной в качестве примера четырехступенчатой конденсаторной батарее (рис. 2) присутствует некоммутируемый базовый конденсатор $C 0$ с емкостью, обеспечивающей гарантированное самовозбуждение АМ с КЗ ротором и номинальный уровень выходного напряжения на холостом ходу до момента подключения питаемой полезной нагрузки контактором $Q F 1$ (рис. 1). Силовая регулируемая часть конденсаторной батареи, состоящая из ряда управляемых конденсаторных стоек, включает коммутируемые конденсаторы C1-C4. Номиналы коммутируемых конденсаторов и число конденсаторных стоек согласуются с параметрами используемой АМ с К3 ротором и подбираются таким образом, чтобы обеспечить дискретное регулирование суммарной емкости конденсаторной батареи с шагом, позволяющим стабилизировать генерируемое напряжение с требуемой точностью. Управление подключенными встречнопараллельно тиристорами VS1-VS8, коммутирующими конденсаторы C1-C4, организуется каналами управления КУ1-КУ4, на которые поступают сигна- лы от системы управления на замыкание конденсаторной стойки. Управляющие импульсы от каналов управления поступают на соответствующие блоки коммутации БК1-БК4, непосредственно обеспечивающие корректный алгоритм коммутации тиристоров. Резисторы $R 1-R 4$, имеющие номинал порядка сотен кОм, применяются для плавного разряда конденсаторов $C 1-C 4$ после полного выключения устройства.

Подключение тиристорами конденсаторов $C 1-C 4$ к статорным обмоткам АМ в произвольные моменты времени может приводить к скачкообразному нарастанию тока. Максимальные броски тока проявляются при коммутации полностью заряженных ранее подключавшихся к статорным обмоткам АМ конденсаторов в моменты времени, когда к тиристорам прикладывается наибольшая разность потенциалов. Высокая скорость нарастания тока в цепи коммутируемого конденсатора может приводить к мгновенному перегреву полупроводниковой структуры тиристора и его выходу из строя, для предотвращения чего применяется блок коммутации (рис. 2).

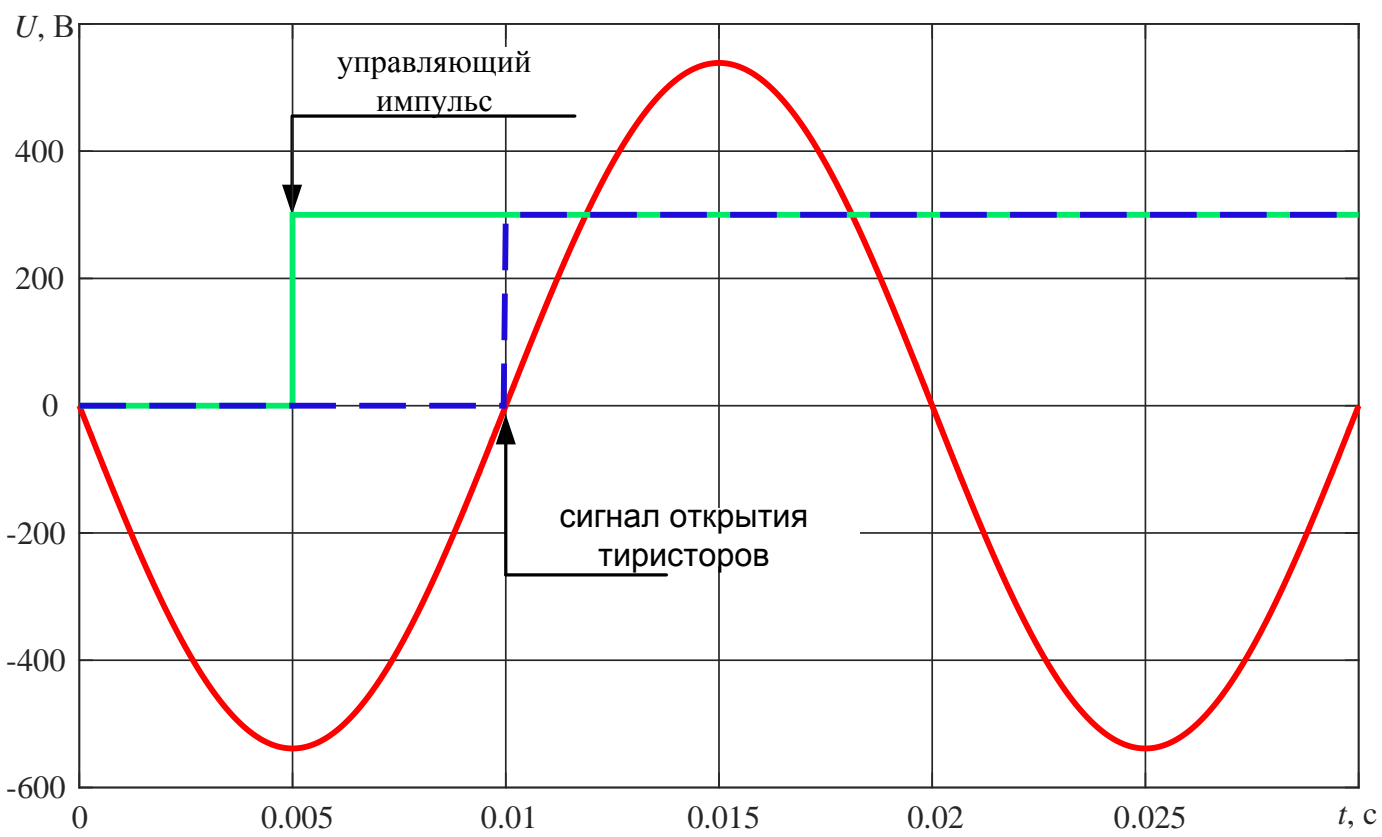

Рис. 3. Временная диаграмма, иллюстрирующая задержку импульса для коммутации тиристорного ключа

Fig. 3. Timing diagram showing the pulse delay of switching the thyristor 
Рис. 3 демонстрирует алгоритм работы блока коммутации (рис. 2) в произвольной конденсаторной стойке при подключении конденсатора, который ранее не задействовался системой управления и был полностью разряжен. Красный синусоидальный сигнал (рис. 3) иллюстрирует линейное напряжение, генерируемое на обмотках АМ с КЗ ротором между двумя произвольными фазами и прикладываемое к подключенным встречно-параллельно тиристорам соответствующей конденсаторной стойки. В произвольный момент времени автоматической системе управления требуется ввести в работу данную конденсаторную стойку, для чего подается управляющий сигнал на соответствующий канал управления (рис. 3). Блок коммутации конденсаторной стойки, который целесообразно реализовать на специализированной оптопаре, оценивает приложенное к тиристорному ключу напряжение. В момент времени, когда приложенное к тиристорному ключу напряжение близко к нулю, блок коммутации выдает сигнал для открытия тиристоров (рис. 3) соответствующей конденсаторной стойки. Коммутация тиристорного ключа происходит при приложении к нему близкой к нулю разности потенциалов, в связи с чем заряд подключенного конденсатора происходит плавно без броска тока и перегрузок использующегося тиристорного ключа. Отключение любой из конденсаторных стоек может производиться в произвольный момент времени и не требует согласования системы управления с блоком коммутации, который при необходимости закрыть тиристорный ключ выдает соответствующий управляющий потенциал без задержек.

Согласно ГОСТ Р 56124.2-2014 «Гибридные электростанции на основе возобновляемых источников энергии, предназначенные для сельской электрификации» не предъявляется жестких требований к качеству напряжения в электросети на основе ВИЭ [25]. Таким образом, система управления, входящая в состав генераторной установки, должна обеспечивать стабилизацию выходного напряжения, формируемого генератором на основе АМ с КЗ ротором, при резком сбросе, набросе или плавном изменении питаемой нагрузки в диапазоне рабочих мощностей с отклонением, не превышающим допустимого уровня для питающих электросетей. Основой системы автоматического управления, обеспечивающей стабилизацию генерируемого напряжения, является предложенный в данной статье алгоритм с применением релейного дискретного регулятора (рис. 3).



Рис. 4. Блок-схема алгоритма стабилизации генерируемого напряжения

Fig. 4. Block diagram of the generated voltage stabilization algorithm 
При достижении валом АМ с КЗ ротором скорости, близкой к номинальной, за счет остаточного намагничения и наличия базовой некоммутируемой емкости $C 0$ (рис. 2) начинается генерация напряжения, часть которого тратится на собственные нужды, а именно на питание системы автоматического управления. При превышении измеряемым фазным напряжением нижней границы рабочей зоны, заданной на уровне $80 \mathrm{~B}$, алгоритм стабилизации вступает в работу. Задачей алгоритма является стабилизация напряжения в зоне допустимых отклонений, в диапазоне от 210 до 230 В. Если измеряемое напряжение опускается ниже зоны допустимых отклонений, происходит увеличение суммарной емкости конденсаторной батареи на 1 мкФ (рис. 2), что позволяет компенсировать провал напряжения. Если измеряемое напряжение поднимается выше зоны допустимых отклонений, соответственно происходит уменьшение суммарной емкости конденсаторной батареи на 1 мкФ (рис. 2), так как текущей уровень подключенной суммарной емкости являлся для системы избыточным.

Предусмотренная в алгоритме задержка на реакцию системы при правильной настройке позволяет избежать избыточного перерегулирования и колебательности стабилизируемого напряжения. В алгоритме также предусмотрен механизм диагностики и оповещения о срыве генерации напряжения, что может быть, к примеру, вызвано токовой перегрузкой в линии питаемой сети.

Исследование процесса стабилизации напряжения генераторной установки на основе имитационной модели

Для проверки принципиальной работоспособности описанных в статье схемотехнических решений и алгоритмов, которые должны обеспечивать работу генератора на базе АМ с КЗ ротором с нестационарным конденсаторным возбуждением, было произведено имитационное моделирования в программной среде MatLab Simulink (рис. 5).

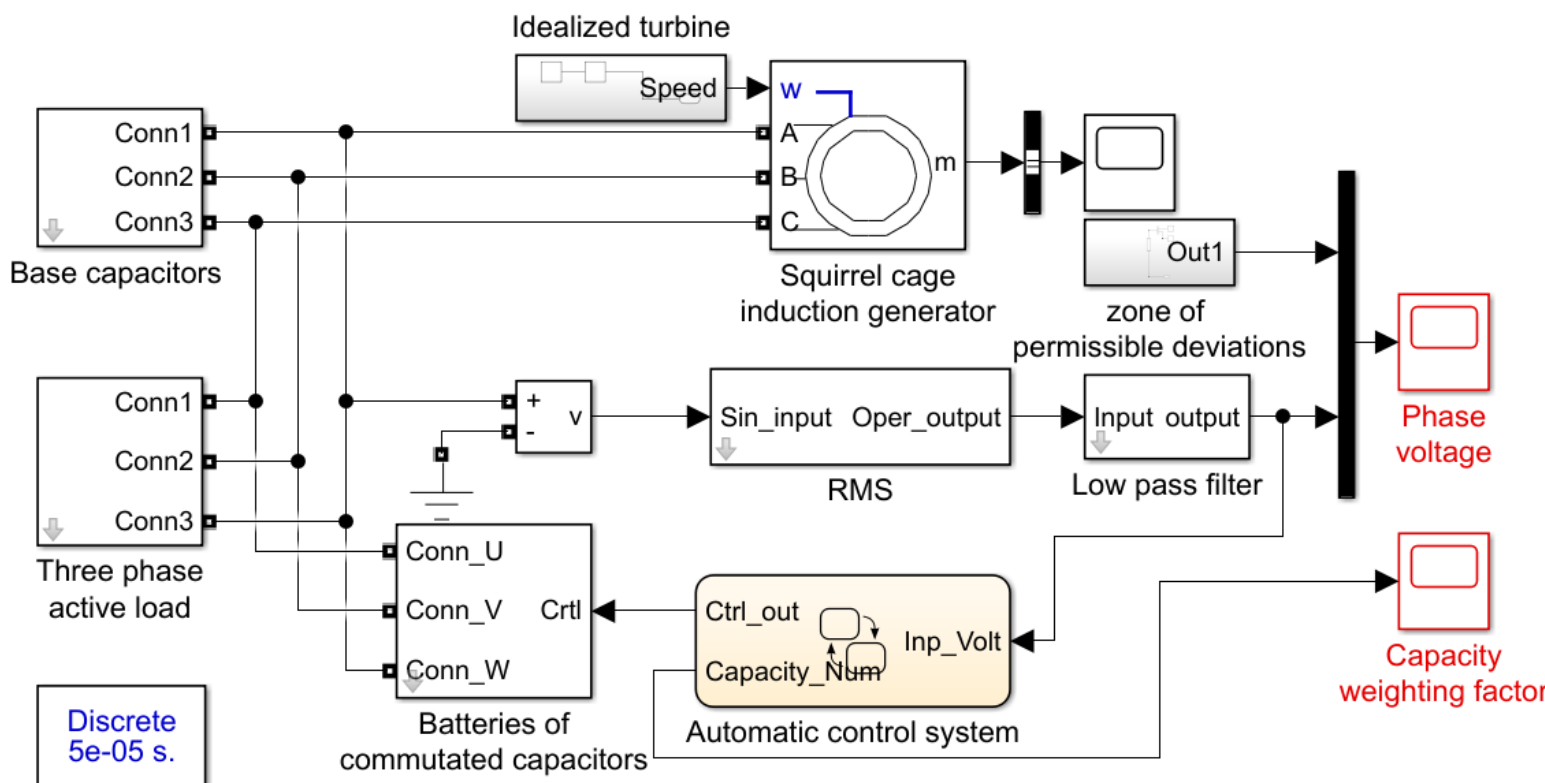

Рис. 5. Имитационная модель электрогенератора на базе АМ с КЗ ротором с нестационарным конденсаторным возбуждением, полезной нагрузкой и автоматической системой управления

Fig. 5. Simulation model of an electric generator based on squirrel-cage induction generator with non-stationary capacitor excitation, load and automatic control system

Имитационная модель электрогенератора (рис. 4) включает АМ с КЗ ротором (Squirrel cage induction generator) с варьируемыми параметрами номинальной мощностью 5,5 кВт, угловая скорость вращения вала которой плавно выводится на номинальный уровень и поддерживается идеализированной моделью турбины (Idealized turbine). Для проявления эффекта самовозбуждения при взаимодействии с базовыми конденсаторами (Base capacitors) с номиналом 15 мкФ в модели асинхронной машины с КЗ ротором требуется задать ненулевые начальные условия. Для генерации корректного уровня напряжения в модели также требуется задать кривую намагничивания AM, близкую к основной кривой намагничивания исследуемой АМ. В модели присутствуют коммутируемые тиристорными ключами конденсаторные батареи
(Batteries of commutated capacitors), конденсаторные стойки которых имеют номиналы 1, 2, 4 и 8 мкФ соответственно. Управление конденсаторными батареями организуется системой автоматического управления (Automatic control system). Для проверки работоспособности механизма стабилизации генерируемого напряжения в модели предусмотрена симметричная трехфазная активная нагрузка, соединенная треугольником, номинальной мощностью 2 кВт, коммутация которой производится идеальными ключами. Сигнал обратной связи в виде мгновенных значений одного из измеряемых фазных напряжений поступает на вход блока расчета действующего уровня напряжения $(R M S)$. Полученный уровень действующего значения фазного напряжения после применения фильтра низких частот первого порядка (Low pass 
filter), постоянная времени которого много меньше электромагнитной постоянной времени АМ с КЗ ротором, поступает на вход системы автоматического управления для формирования управляющих воздействий.

Основным параметром системы автоматического управления, требующим подбора и определяющим быстроту реакции системы на возмущающее воздействие, а именно изменение питаемой генераторной установкой нагрузки, является период дискретизации. Результаты имитационного моделирования генераторной установки при задании различного периода дискретизации САУ в различных режимах работы представлены на рис. 6 .

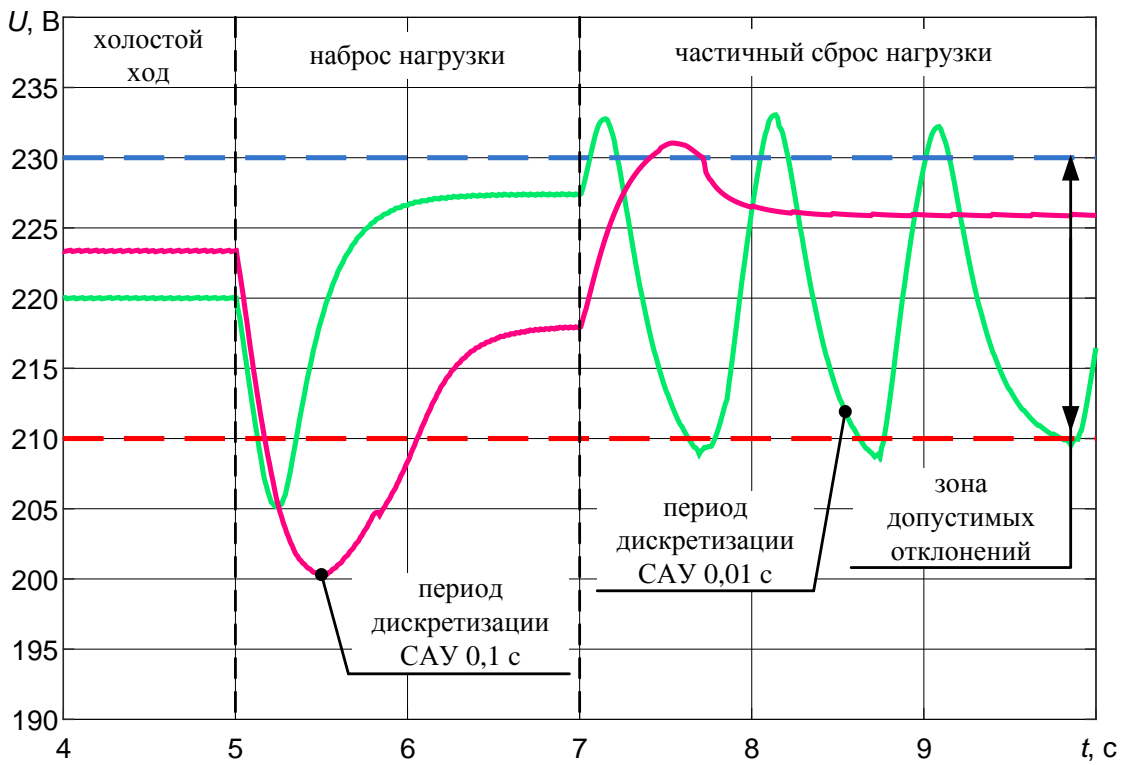

Рис. 6. Временные диаграммы фазного напряжения генераторной установки в различных режимах работы при варьировании периода дискретизации системы автоматического управления

Fig. 6. Timing diagrams of the phase voltage of the generator bench in various operating modes with varying sampling period of the automatic control system

Задание периода дискретизации САУ, организующей управление коммутируемыми конденсаторными батареями (рис. 2), равного 0,1 с, обеспечивает стабилизацию напряжения (рис. 6) генераторной установки в режимах холостого хода, наброса и частичного сброса подключаемой активной симметричной нагрузки в зоне допустимых отклонений. Задание периода дискретизации САУ, равного 0,01 с, обеспечивает более высокую скорость реакции на изменение питаемой нагрузки, однако приводит к избыточной колебательности контролируемого напряжения.

Полученные в результате моделирования при задании периода дискретизации САУ 0,1 с итоговые графики фазного напряжения генераторной установки (рис. 7, б) и суммарной емкости (рис. $7, a$ ) одной из конденсаторных батарей без учета емкости базового конденсатора представлены ниже.

Модель генераторной установки демонстрирует успешную стабилизацию фазного напряжения (рис. $7,6)$, отклонение которого не превышает 20 В относительно номинальных $220 \mathrm{~B}$, при набросе и сбросе полной активной симметричной нагрузки мощностью 2 кВт. При этом заданный период дискретизации САУ и выбранные уставки зоны допустимых отклонений, обеспечивающей релейную регулировочную характеристику, позволяют уменьшать число коммутаций тиристорных ключей при регулировании суммарной емкости конденсаторной батареи, снижая нагрузку на силовую часть устройства.

\section{Исследование процесса стабилизации напряжения} генераторной установки на экспериментальной установке

Натурные испытания системы стабилизации напряжения производились на макете генераторной установки на базе АМ с КЗ ротором с нестационарным конденсаторным возбуждением (рис. 8).

В качестве генератора применялась АМ с К3 ротором АДM100L2 (рис. 8) номинальной мощностью 5,5 кВт с синхронной скоростью 3000 об/мин. Имитацию работы турбины генераторной установки осуществлял дизельный двигатель KM186FAG, приводя в движение вал АМ, обеспечивая уровень угловой скорости, близкий к номинальному значению. В качестве резервного источника питания применялся аккумулятор. Каждая из трех конденсаторных батарей, соединенных треугольником (рис. 1), включала четыре коммутируемые конденсаторные стойки с номиналами 1, 2, 4 и 8 мкФ соответственно, а также базовый конденсатор емкостью 15 мкФ. Выбранное число ступеней обеспечило шаг регулирования суммарной емкости конденсаторной батареи, без учета емкости базового конденсатора, в 1 мкФ в диапазоне от 1 до 15 мкФ. В качестве тиристорного ключа, осуществляющего коммутацию в конденсаторных батареях, применялся SK45UT12 с номинальным током 45 А, номинальным напряжением для силового канала 1200 В. Блок коммутации (рис. 2) был реализован на основе оптопары МОС $3082 M$ с симисторным выходом. Система автоматического управления была реализована на основе микроконтроллера STM32F103C8T6. 

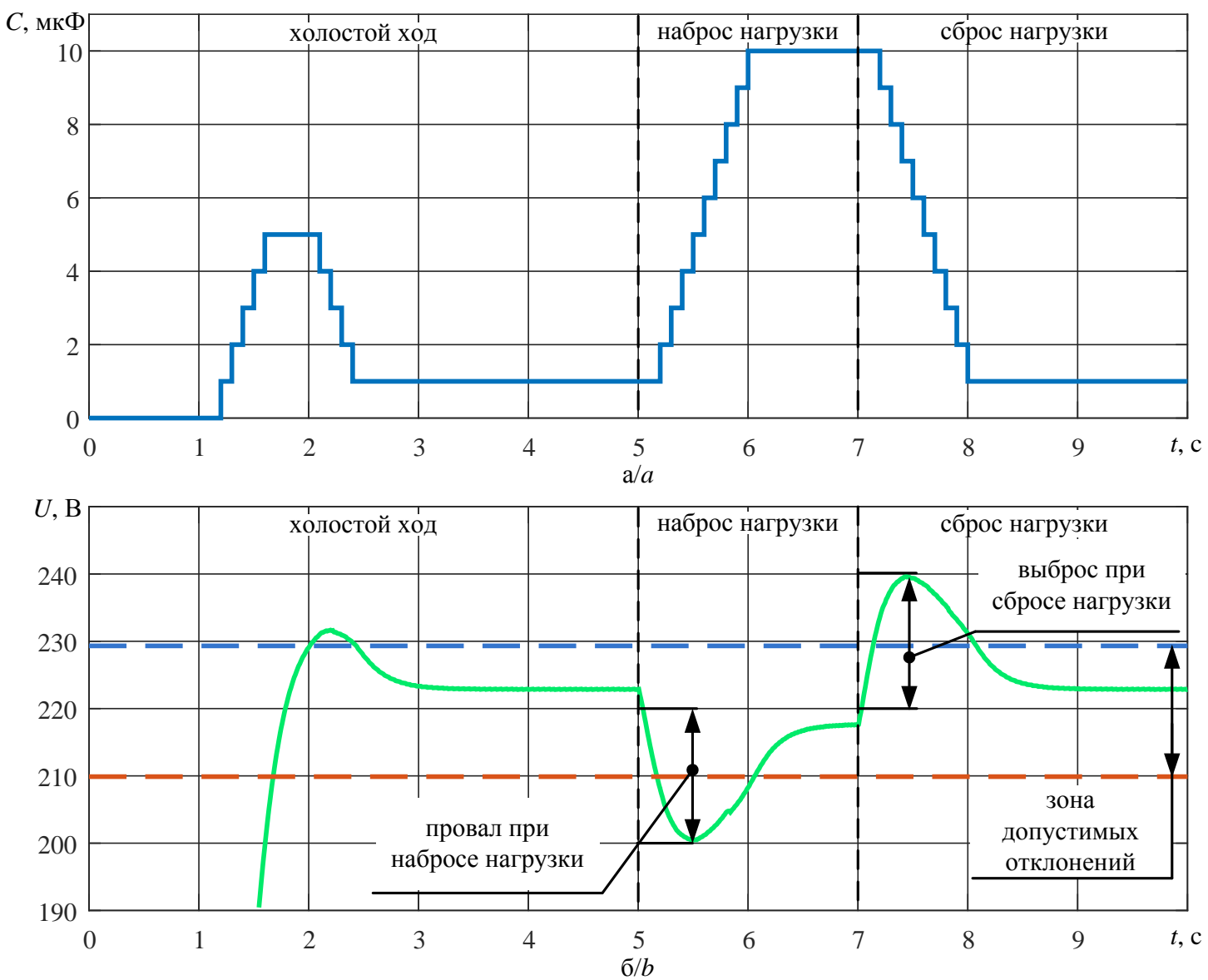

Рис. 7. Временные диаграммы регулируемой суммарной емкости конденсаторной батареи без учета емкости базового конденсатора (а) и стабилизуемого фазного напряжения (б) генераторной установки в различных режимах работы, полученные при моделировании

Fig. 7. Timing diagrams of the controlled total capacity of the capacitor bank without base capacity (a) and the stabilized phase voltage $(b)$ of the generator bench in various operating modes after simulation

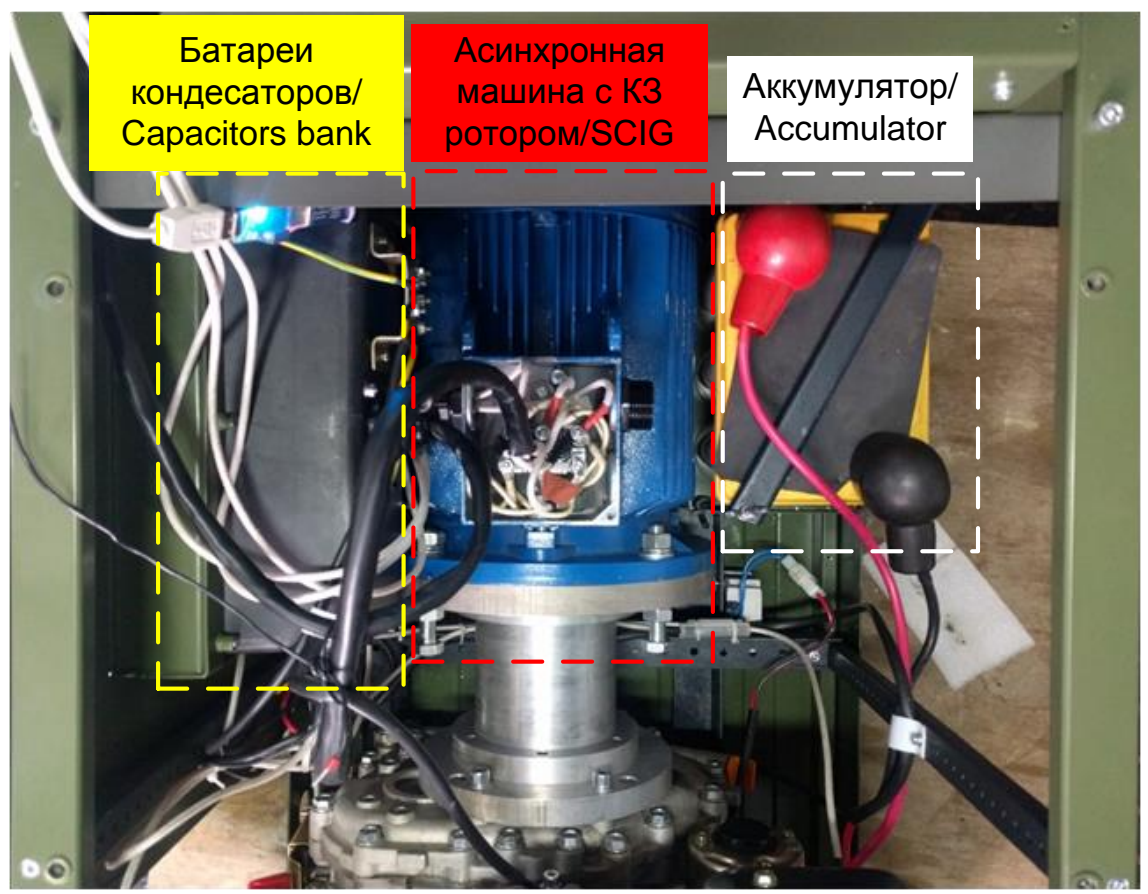

Pис. 8. Внешний вид макета генераторной установки на базе АМ с КЗ ротором с нестационарным конденсаторным возбуждением

Fig. 8. Photo of the squirrel-cage induction generator bench with non-stationary capacitor excitation 
Для подтверждения возможности регулирования напряжения, генерируемого АМ с КЗ ротором, при различной мощности питаемой активной симметрич- ной нагрузки были построены семейства вольтфарадных и герц-фарадных регулировочных характеристик генераторной установки (рис. 9).

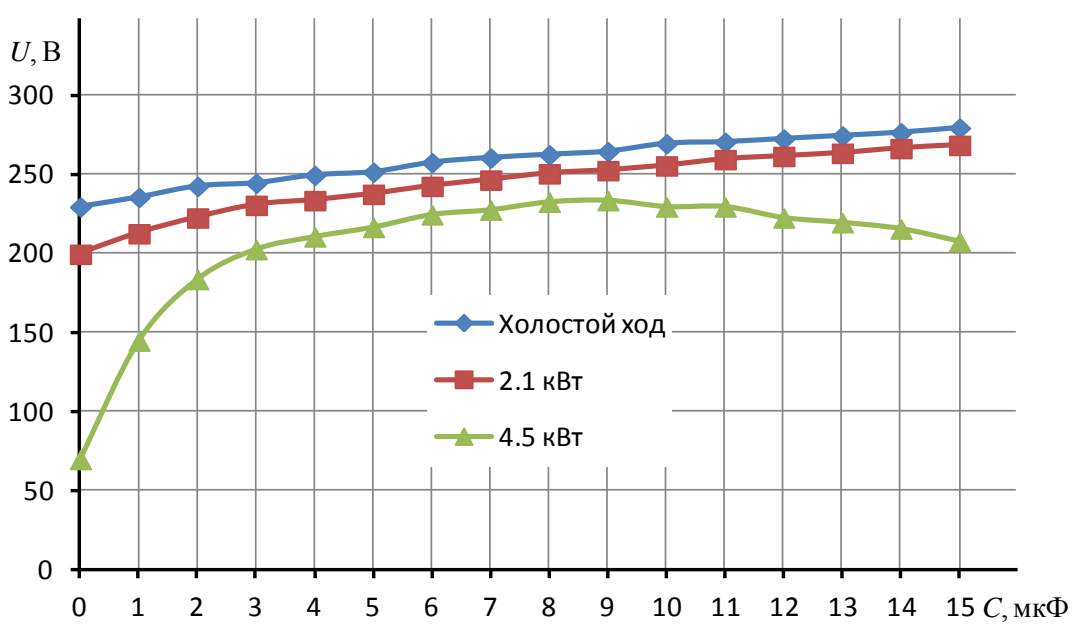

Pис. 9. Вольт-фарадные регулировочные характеристики при различной номинальной мощности питаемой активной симметричной нагрузки без учета емкости базового конденсатора

Fig. 9. Voltage-capacitance control characteristics at different rated power of the supplied active balanced load without base capacity

Вольт-фарадные регулировочные характеристики при работе на холостом ходу и на активную нагрузку с номинальным значением потребляемой мощности 2,1 кВт имеют практически линейных характер, что позволяет реализовать систему стабилизации фазного напряжения (рис. 4), малочувствительную к изменению нагрузки в диапазоне $0 \ldots 2,1$ кВт. При работе на нагрузку с номинальным значением потребляемой мощности 4,5 кВт в диапазоне дополнительных регу- лируемых емкостей от $0 \ldots .3$ мкФ наблюдается явная нелинейность характеристики, связанная с недовозбуждением, что объясняется тем, что АМ с КЗ ротором представляет собой сильно нелинейный объект в условиях значительной перегрузки. В диапазоне от 3 ...9 мкФ характеристика носит линейно нарастающий характер. В диапазоне от $10 \ldots 15$ мкФ наблюдается спадающий участок, объясняемый работой приводного дизельного двигателя в перегруженном режиме.

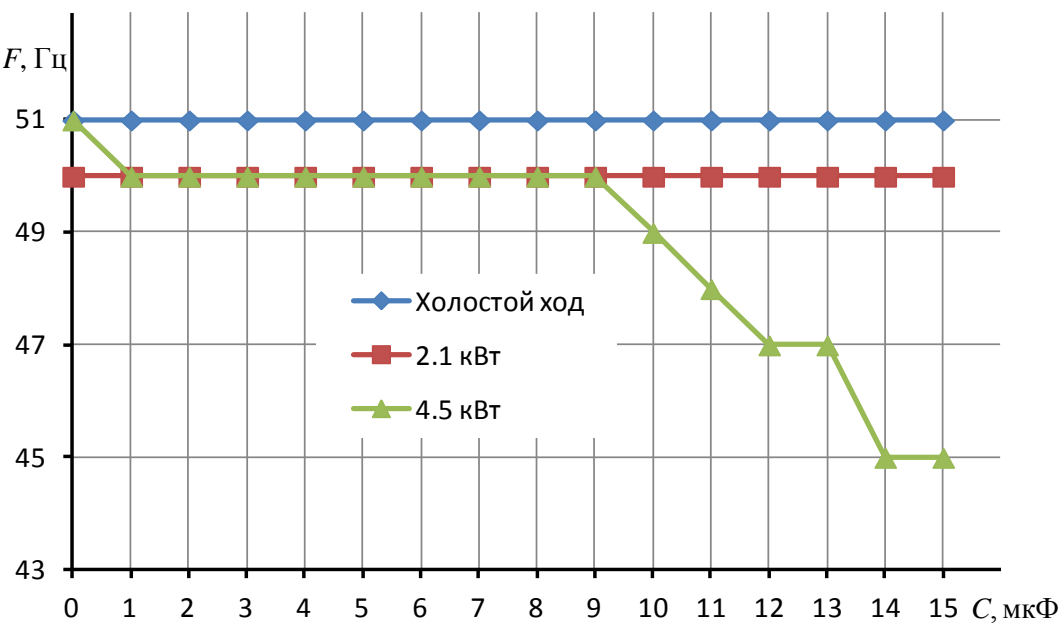

Рис. 10. Гери-фарадные регулировочные характеристики при различной мощности питаемой активной симметричной нагрузки

Fig. 10. Frequency-capacitance control characteristics at different power of the supplied active balanced load

Герц-фарадные регулировочные характеристики при работе на холостом ходу и на активную нагрузку с номинальным значением потребляемой мощности 2,1 кВт имеют практически линейных характер, демонстрируя отсутствие зависимости частоты переменного тока генератора от емкости конденсаторной батареи без учета базового конденсатора. В случае предельной для данной установки мощности нагрузки с номинальным значением 4,5 кВт для диапазона емкостей $9 . . .15$ мкФ дополнительных коммутируемых конденсаторов наблюдается пропорциональное снижение частоты переменного тока генератора с 50 до 45 Гц. Этот эффект объясняется тем, что данный генератор является источником ограниченной мощности. 



Рис. 11. Временные диаграммы регулируемой суммарной емкости конденсаторной батареи (а) и стабилизуемого фазного напряжения (б) генераторной установки в различных режимах работы, полученные в результате эксперимента

Fig. 11. Timing diagrams of the controlled total capacity of the capacitor bank (a) and the stabilized phase voltage (b) of the generator bench in various operating modes after the experiment

Результаты тестирования алгоритма управления возбуждением генераторной установки, синтезированного на основе имитационной модели (рис. 4-7) и реализованного в программном обеспечении микроконтроллера, представлены на рис. $11, a, \sigma$.

Система, изначально запущенная на холостом ходу, имела напряжение близкое к 220 В (рис. $11, a$, б). При этом зона допустимых отклонений напряжения была задана $220 \pm 15$ В, а период дискретизации САУ равнялся 0,1 с. На второй секунде была подключена активная нагрузка номинальной мощностью 2,1 кВт, что вызвало динамический провал напряжения не более $20 \%$ с последующей стабилизацией напряжения в течение не более одной секунды, при этом статическая ошибка составляла не более 10 В. После сброса нагрузки динамический выброс напряжения не превысил $20 \%$ с последующей стабилизацией в течение не более одной секунды.

\section{Заключение}

Анализ экспериментальных зависимостей переходных процессов выходного напряжения генератора с нестационарным конденсаторным возбуждением доказывает работоспособность предложенного метода стабилизации напряжения АМ с КЗ ротором.

Показатели качества электроэнергии, вырабатываемой генераторной установкой на базе АМ с КЗ ротором с нестационарным конденсаторным возбуждением, соответствуют требованиям ГОСТ Р 56124.2-2014 «Гибридные электростанции на основе возобновляемых источников энергии, предназначенные для сельской электрификации» при работе на симметричную активную нагрузку, что делает возможным ее применение в составе ВЭУ и микро-ГЭС.

Дальнейшим развитием проведенных исследований может стать анализ поведения представленной системы при работе на другие типы нагрузок, в том числе несимметричные и нелинейные. 


\section{СПИСОК ЛИТЕРАТУРЫ}

1. Безруких П.П., Безруких П.П. мл. Ветроэнергетика. Вымыслы и факты. Ответы на 100 вопросов. - М.: Институт устойчивого развития Общественной палаты Российской Федерации/Центр экологической политики России, 2011. - 74 с.

2. Власов В.К. Ветродвигатели. Теория и практика. - М.: РИЦ «Техносфера», 2020. - 227 c.

3. Papera L.M. Microhydro power plant future energy source. Saarbrücken, Germany: LAP LAMBERT Academic Publishing, 2017. $-56 \mathrm{p}$.

4. Возобновляемая энергия. Гидроэлектростанции России. Справочник / М.И. Дворецкая, А.П. Жданова, О.Г. Лушников, И.В. Слива / под общей ред. к. т. н., проф. В.В. Берлина. СПб.: Изд-во Политехн. ун-та, 2018. - 224 с.

5. Optimization potential analysis of micro-hydro power plan (MHPP) from river with low head / R. Marliansyah, D.N. Putri, A. Khootama, H. Hermansyah // Energy Procedia. - 2018. V. 153. - P. 74-79

6. Abolvafaei M., Ganjefar S. Maximum power extraction from fractional order doubly fed induction generator based wind turbines using homotopy singular perturbation method // International Journal of Electrical Power \& Energy Systems. 2020. - V. 119. - P. 1-17.

7. Dinh-Chung Phan, Yamamoto S. Rotor speed control of doubly fed induction generator wind turbines using adaptive maximum power point tracking // Energy. - 2016. - V. 111. - P. 377-388.

8. Tapia R., Medina A. Doubly-fed wind turbine generator control: a bond graph approach // Simulation Modelling Practice and Theory. - 2015. - V. 53 - - P. 149-166.

9. Николаев В.Г., Харченко В.В. Перспективы и проблемы развития в России ветродизельных энергетических комплексов // Энергетик. - 2016. - № 3. - С. 5-7.

10. Альтернативные источники: энергия ветра, плюсы и минусы // Энерго.Хаус. 2020. URL: https://energo.house/veter/energiyavetra.html (дата обращения 20.11.2020).

11. Севастьянов В.В., Сапьян Е.С. Ветроэнергетические ресурсы центральных районов Республики Алтай // Известия Томского политехнического университета. Инжиниринг георесурсов. 2019. - T. 330. - № 2. - С. 55-64.

12. Ощепкова Я.О., Киушкина В.Р. Кластерный анализ потенциала возобновляемых источников энергии в Республики Саха (Якутия) // Интернет-журнал «НАУКОВЕДЕНИЕ». - 2014. T. 4. - № 23. - C. 1-19.

13. Киушкина В.Р. Повышение энергетической безопасности децентрализованных зон электроснабжения регионов северных территорий и арктических зон (на примере Республики Саха (Якутия)): автореф. дис. ... д-р. техн. наук. - Красноярск, 2019. - 38 с.

\section{Информация об авторах}

Буньков Д.С., инженер-программист, ООО «Элетим».

Глазырин A.C., доктор технических наук, профессор отделения электроэнергетики и электротехники Инженерной школы энергетики Национального исследовательского Томского политехнического университета; профессор Института нефти и газа Югорского государственного университета.

Боловин $\boldsymbol{E}$.B., кандидат технических наук, старший преподаватель отделения электроэнергетики и электротехники Инженерной школы энергетики Национального исследовательского Томского политехнического университета.

Крохта Ю.В., инженер-программист, ООО «Элетим».

Баннов Д.М., инженер кафедры электрических станций Электротехнического факультета Самарского государственного технического университета.

Ковалев В.3., доктор технических наук, профессор Института нефти и газа Югорского государственного университета.

Хамитов Р.Н., доктор технических наук, доцент, профессор кафедры электрической техники Омского государственного технического университета; профессор кафедры электроэнергетики Тюменского индустриального университета.

Кладиев $\boldsymbol{C . H . , ~ к а н д и д а т ~ т е х н и ч е с к и х ~ н а у к , ~ д о ц е н т ~ о т д е л е н и я ~ э л е к т р о э н е р г е т и к и ~ и ~ э л е к т р о т е х н и к и ~ И н ж е н е р - ~}$ ной школы энергетики Национального исследовательского Томского политехнического университета.

Ланграф С.В., кандидат технических наук, руководитель сектора разработки встроенного ПО, ООО «НПФ Мехатроника-Про».

Леонов А.П., кандидат технических наук, доцент отделения электроэнергетики и электротехники Инженерной школы энергетики Национального исследовательского Томского политехнического университета. 
UDC 621.313 .332

\title{
RESEARCH OF A SQUIRREL-CAGE INDUCTION GENERATOR FOR RENEWABLE ENERGY WITH NON-STATIONARY CAPACITOR EXCITATION
}

\author{
Dmitriy S. Bunkov 1 , \\ bunkovds@tpu.ru
}

\author{
Alexander S. Glazyrin²,3, \\ asglazyrin@tpu.ru
}

\author{
Evgeniy V. Bolovin², \\ orange@tpu.ru
}

Yuriy V. Krokhta1, yuriy.krokhta@gmail.com

\author{
Dmitriy M. Bannov 4 , \\ bannov.dm@samgtu.ru
}

1 Eletim Ltd, 161A, Altayskaya street, Tomsk, 634021, Russia.

2 National Research Tomsk Polytechnic University, 30, Lenin avenue, Tomsk, 634050, Russia.

3 Yugra State University, 16, Chekhov street, Khanty-Mansiysk, 628012, Russia.

${ }^{4}$ Samara State Technical University, 244, Molodogvardeyskaya street, Samara, 443100, Russia.

5 Omsk State Technical University, 11, Mira avenue, Omsk, 644050, Russia.

6 Tyumen Industrial University, 38 , Volodarsky street, Tyumen, 625000, Russia.

7 NPF Mechatronica-Pro LTD, 119E, Frunze avenue, Tomsk, 634021, Russia.

\author{
Vladimir Z. Kovalev ${ }^{3}$, \\ vz_kovalev@mail.ru
}

\author{
Rustam N. Khamitov5,6, \\ apple_27@mail.ru
}

\author{
Sergey N. Kladiev2 \\ kladiev@tpu.ru
}

\author{
Sergey V. Langraf ${ }^{7}$ \\ sergeylgrf@gmail.com
}

\author{
Andrey P. Leonov², \\ leonov_ap@tpu.ru
}

\begin{abstract}
The relevance. The share of electricity generated by installations based on renewable energy is constantly growing, and therefore the need for the development of power supply systems and automatic control of electrical machines underlying wind and hydro generators is still relevant. As part of such alternating current generator sets, synchronous electric machines, induction generator with phase and squirrel-cage rotor are used. Frequency converters installed in the stator and rotor circuits of induction generators make it possible to control their excitation, however, to start generation, additional external power sources must be used. Due to the residual magnetization in the magnetic circuit, it is possible to ensure the process of guaranteed self-excitation of the induction generator by connecting the capacitor banks to the electrical circuit of its stator without using an additional external power source. The proposed method of non-stationary capacitor excitation makes it possible to ensure the adaptation of the generator set to the change in operating modes under conditions of decentralized power supply.

The main aim of the research is to study the proposed voltage stabilization system of squirrel-cage induction generator with a short-circuit rotor with variable capacitor excitation as part of a renewable energy source.

Methods. To achieve the goal of the study, theoretical and experimental research methods were used. Theoretical research methods include the theory of differential equations, methods for the numerical solution of ordinary differential equations, the theory of electric drives, the theory of electrical machines, numerical methods for approximating data. The basis of experimental research is testing an induction generator with non-stationary capacitor excitation on a developed test bench in order to obtain load characteristics and oscillograms of voltages on the stator windings self-excitation of squirrel-cage induction generator rotor in different operating modes.

Results. An original experimental setup with a unit imitating the operation of a turbine, an electromechanical energy converter in the form of squirrel-cage induction generator with non-stationary capacitor excitation has been developed and manufactured. The control system is made in the form of a galvanically isolated module integrated with the power unit in a moisture-resistant design using a wireless Bluetooth communication interface. A method for switching capacitor banks using thyristors as a controlled switch with two-way conduction is described and tested. The analysis of the obtained load characteristics shows the fundamental possibility of ensuring a guaranteed generation of electricity with voltage stabilization within the limits of load power variation. The system of automatic switching of capacitors with a capacitance varying depending on the power consumption applied as part of the experimental setup made it possible to provide an acceptable time for dynamic response to a disturbing effect under nonstationary load.
\end{abstract}

\section{Key words:}

Renewable energy sources, induction generator, squirrel-cage rotor, non-stationary capacitor excitation, decentralized power supply. 


\section{REFERENCES}

1. Bezrukih P.P., Bezrukih P.P Jr. Vetroenergetika. Vymysly $i$ fakty. Otvety na 100 voprosov [Wind power. Fiction and facts. Answers to 100 questions]. Moscow, Institute of Sustainable Development of the Public Chamber of the Russian Federation/Center environmental policy of Russia Publ., 2011. 74 p.

2. Vlasov V.K. Vetrodvigatili. Teoria i praktika [Wind turbines. Theory and practice]. Moscow, Tekhnosfera Publ. center, 2020. $227 \mathrm{p}$.

3. Papera L.M. Microhydro power plant future energy source. Saarbrücken, Germany, LAP LAMBERT Academic Publ., 2017. 56 p.

4. Dvortskaya M.I., Zhdanova A.P., Lushnikov O.G., Sliva I.V Vozobnovlyamaya energiya. Gidroelectostantsii Rossii. Spravochnik [Renewable energy. Hydroelectric power plants of Russia. Directory]. St. Petersburg, Polytechnic university Publ., 2018. 224 p.

5. Marliansyah R., Putri D.N., Khootama A., Hermansyah H. Optimization potential analysis of micro-hydro power plant (MHPP) from river with low head. Energy Procedia, 2018, vol. 153 pp. 74-79

6. Abolvafaei M., Ganjefar S. Maximum power extraction from fractional order doubly fed induction generator based wind turbines using homotopy singular perturbation method. International Journal of Electrical Power \& Energy Systems, 2020, vol. 119, pp. 1-17.

7. Dinh-Chung Phan, Yamamoto S. Rotor speed control of doubly fed induction generator wind turbines using adaptive maximum power point tracking. Energy, 2016, vol. 111, pp. 377-388.

8. Tapia R., Medina A. Doubly-fed wind turbine generator control: a bond graph approach Simulation Modelling Practice and Theory, 2015, vol. 53, pp. 149-166.

9. Nikolaev V.G., Kharchenko V.V. Perspektivy i problemy razvitiya v Rossii vetrodizelnykh energetiheskikh kompleksov [Prospects and problems of development of wind-diesel power complexes in Russia]. Energetik, 2016, no. 3, pp. 5-7.

10. Alternativnye istochniki: energiya vetra, plyusy i minusy [Alternative sources: wind energy, pros and cons]. Energo.Khaus. 2020 Available at: https://energo.house/veter/energiya-vetra.html (accessed 20 November 2020)

11. Sevastyanov V.V., Sapian E.S. Windpower resources of central regions of the Altai Republic. Bulletin of the Tomsk Polytechnic University. Geo Assets Engineering, 2019, vol. 330, no. 2, pp. 55-64 In Rus.

12. Oshchepkova Ya.O., Kiushkina V.R. Klasterny analiz potentsiala vozobnovlyaemykh istochnikov energii $\mathrm{V}$ Respubliki Sakha (Yakutiya) [Cluster analysis of the potential of renewable energy sources in the Republic of Sakha (Yakutia)]. Online magazine «Science», 2014, vol. 4, no. 23, pp. 1-19.

13. Kiushkina V.R. Povyshenie energeticheskoy bezopasnosti detsen tralizovannykh zon elektrosnabzheniya regionov severnykh territoriy $i$ arkticheskikh zon (na primere Respubliki Sakha (Yakutiya)). Avtoreferat Dis. Dokt. nauk [Improving the energy security of decentralized power supply zones in the regions of the northern territories and arctic zones (on the example of the Republic of Sakha (Yakutia)). Dr. Diss. Abstract]. Krasnoyarsk, 2019. 38 p.
14. Sevastyanova L.M., Nikolchenko Yu.N. Potentsialnye vetro- i gelioenergeticheskie resursy v altayskom krae [Potential wind and solar energy resources in the Altai Territory]. Tomsk State University Journal, 2012, no. 365, pp.187-193.

15. Energeticheskaya strategiya Rossiyskoy Federatsii na period do 2035 goda [Energy strategy of the Russian Federation for the period up to 2035]. Moscow, Ministry of energy of Russian Federation, $2020.93 \mathrm{p}$.

16. Krasnov V.G., Kasatkina E.V., Kalinina M.V. Ekonomicheskaya tselesoobraznost izyskaniy ratsionalnykh konstruktsiy mikro-GES [Economic feasibility of researching rational structures of microhydroelectric power plants]. Innovation and investment, 2019, no. 2, pp. 166-169.

17. Vershinina I.P. Evaluation of energy potential of small rivers of the Tom. Bulletin of the Tomsk Polytechnic University. Geo Assets Engineering, 2016, vol. 327, no. 3, pp. 111-118. In Rus.

18. Avilov V.D., Serkova L.E. Malaya gidroenergetika i energeticheskaya stratagiya Sibirskogo regiona [Small hydropower and energy strategy of the Siberian region]. Russia's national priorities, 2009, no. 1, pp. 95-113.

19. Gil-González W., Montoya O.D., Garces A. Standard passivitybased control for multi-hydro-turbine governing systems with surge tank Author links open overlay. Applied Mathematical Modelling, 2020, vol. 79, pp. 1-17.

20. Yin X., Cheng L., Wang X., Lu J., Qin H. Optimization for hydrophotovoltaic-wind power generation system based on modified version of multi-objective whale optimization algorithm. Energy Procedia, 2019, vol. 158, pp. 6208-6216.

21. Lukutin B.V., Obukhov S.G., Shandarova E.B. Sposoby povysheniya kachestva vykhodnogo napryazheniya mikrogidroelectostantsii s tiristornym avtoballastom [Ways to improve the quality of the output voltage of a microhydroelectric power plant with a thyristor autoballast]. Industrial power engineering, 2000, no. 8, pp. 49-52.

22. Hossain Md.M., Ali Mohd.H. Future research directions for the wind turbine generator system. Renewable and Sustainable Energy Reviews, 2015, vol. 49, pp. 481-489.

23. Volkov A.G. Mnogozonnye elektronnye konvertory dlya avtonomnykh sistem generirovaniya electricheskoy energii. Dis. Kand. nauk [Multi-zone electronic converters for autonomous power generation systems. Cand. Diss.]. Novosibirsk, 2016. 192 p.

24. Mikhalchenko S.G., Russkin V.A., Semenov S.M., Orlyanskiy I.P., Hala'sz S. Construction of adaptive algorithm of power extreme control in solar energy system. Bulletin of the Tomsk Polytechnic University. Geo Assets Engineering, 2018, vol. 329, no. 3, pp. 102-112. In Rus.

25. GOST R 56124.2-2014. Gibridnye electrostantsii na osnove vozobnovlyaemykh istochnikov energii, prednaznachennykh dlya selskoy mestnosti [State Standard R 56124.2-2014. Renewable hybrid power plants for rural electrification]. Moscow, StandardInform Publ., 2016. 49 p.

Received: 3 December 2020.

\section{Information about the authors}

Dmitriy S. Bunkov, software engineer, Eletim Ltd.

Alexander S. Glazyrin, Dr. Sc., professor, National Research Tomsk Polytechnic University; professor, Yugra State University.

Evgeniy V. Bolovin, Cand. Sc, senior lecture, National Research Tomsk Polytechnic University.

Yuriy V. Krokhta, software engineer, Eletim Ltd.

Dmitriy M. Bannov, engineer, Samara State Technical University.

Vladimir Z. Kovalev, Dr. Sc., professor, Yugra State University.

Rustam N. Khamitov, Dr. Sc., professor, Omsk State Technical University; professor, Tyumen Industrial University.

Sergey N. Kladiev, Cand. Sc., associate professor, National Research Tomsk Polytechnic University.

Sergey $V$. Langraf, head of the embedded software development sector, NPF Mechatronica-Pro Ltd.

Andrey P. Leonov, Cand. Sc., associate professor, National Research Tomsk Polytechnic University. 